\title{
Grammaire matérielle et erreurs de catégories
}

Par BRUNO LECLERCQ

Université de Liège

\section{L'idée husserlienne d'une grammaire pure logique}

L'idée d'une morphologie pure des significations que développe Husserl dans la quatrième de ses Recherches logiques tient dans le projet d'identifier, d'une part, les « catégories essentielles de la signification » et, d'autre part, fondées en elles, une multiplicité de lois de leurs combinaisons en significations unitaires ${ }^{1}$. "Faisant abstraction de la validité objective (de la vérité ou encore de l'objectivité réelles ou formelles) des significations », dit Husserl, ces «lois de la signification » sont des « lois a priori qui régissent la sphère des complexions de significations et qui ont pour fonction de séparer en elles sens et non-sens $»^{2}$. De telles lois «morphologiques », précise-t-il, ne sont

pas encore ce qu'on appelle des lois logiques au sens fort du mot; elles donnent à la logique pure les formes possibles de la signification, c'est-à-dire les formes a priori de significations complexes, ayant une unité de sens, dont

\footnotetext{
${ }^{1}$ Le présent texte reconfigure, développe et prolonge un certain nombre de thématiques abordées une première fois dans l'article "Catégories sémantiques et catégories syntaxiques: relecture critique des Recherches logiques par la philosophie analytique », dans A. Dewalque, B. Leclercq, D. Seron (éds.), Catégories logiques et catégories ontologiques, Liège, Presses Universitaires de Liège - Sciences Humaines, 2011, p. 149-188. Plusieurs des passages du présent texte sont repris du précédent article.

${ }^{2}$ E. Husserl, Recherches logiques, IV (1901), Paris, P.U.F., Épiméthée, 1959-1963, tome II, Intro, p. 85.
} 
ensuite les « lois logiques » au sens fort du mot régissent la vérité "formelle » ou «l'objectivité » ${ }^{1}$.

Avant même la question de la validité des énoncés et des éventuels rapports de contradiction ou de conséquence qu'ils entretiennent, une discipline doit établir la liste des différentes composantes fondamentales de la signification et les lois de leur composition en des touts dotés d' ' unité de sens » ${ }^{2}$. Présupposées par les lois pures logiques, qui «préviennent le contresens formel ou analytique, l'absurdité formelle », les lois pures morphologiques «préviennent le non-sens » et « déterminent ce qu'exige la simple unité $d u$ sens, c'est-à-dire les formes a priori selon lesquelles des significations appartenant aux diverses catégories de significations se réunissent en une seule signification, au lieu de produire un non-sens chaotique $»^{3}$.

C'est en fait sur le fondement même de cette distinction entre sens et non-sens - distinction qui est donc préalable à celle qui s'opère, au sein de la sphère du sens, entre jugement consistants et jugements contradictoires - , que Husserl détermine la notion de " catégorie de signification » à partir d'un principe d'intersubstituabilité "salva significatione »; deux significations appartiennent à une même catégorie si elles peuvent être substituées l'une à l'autre sans affecter la prétention à l'unité de sens du tout dans lequel elles s'insèrent :

L'expression cet arbre est vert possède une unité de signification. Si nous passons, en formalisant, de cette signification donnée à la forme de signification pure correspondante, à la «forme propositionnelle», nous obtenons ce $S$ est $p[\ldots]$. Il est dès lors clair que la matérialisation, pour ainsi dire, de cette forme, sa particularisation en propositions déterminées, est possible d'une infinité de manières, que cependant nous ne sommes pas ici pleinement libres, mais que nous devons nous tenir à l'intérieur de limites déterminées. On ne peut substituer à la variable $S$ pas plus qu'à $p$ n'importe quelle signification. Nous pouvons sans doute, dans le cadre de cette forme, remplacer notre exemple : cet arbre est vert, par cet or, ce nombre algébrique $n$, ce corbeau bleu, etc. est vert, bref, nous pouvons placer ici n'importe quelle matière nominale, en prenant ce mot dans un sens large, et pareillement, nous pouvons, bien entendu, mettre à la place de $p$ n'importe quelle matière adjective: nous obtenons alors toujours une signification douée d'unité de sens, à savoir une proposition indépendante de la forme

${ }^{1}$ Ibid.

${ }^{2}$ Ibid., p. 85-86. Cf. aussi « Prolégomènes à la logique pure » (1900), in Recherches logiques, op. cit., tome I, §67, p. 267-270.

${ }^{3}$ E. Husserl, Recherches logiques, IV, op. cit., tome II, Intro, p. 85-86. 
prédonnée. Mais, dès que nous ne respectons pas les catégories des matières de signification, l'unité de sens disparaît. Là où il y a une matière nominale, on peut mettre la matière nominale que l'on veut, mais non pas une matière adjective, ou relationnelle, ni une matière propositionnelle tout entière ; mais là où il y a une matière de l'une de ces catégories, on peut toujours la remplacer par une nouvelle matière de la même catégorie et non d'une autre. Cela s'applique à n'importe quel genre de significations, quelque compliquée que puisse être leur forme ${ }^{1}$.

Cette notion de «catégorie de signification » est clairement un élément central de la morphologie pure des significations esquissée dans la quatrième Recherche logique. Les lois de la composition du sens semblent en effet reposer entièrement sur la répartition des significations en catégories morphologiques :

Quand on substitue librement les unes aux autres des matières à l'intérieur de leur catégorie, il peut en résulter des significations (des propositions entières ou des membres possibles de propositions) fausses, absurdes ou risibles, mais il doit nécessairement en résulter des significations unitaires ou des expressions grammaticales dont le sens peut être réalisé comme unité de sens. Dès que nous transgressons les frontières des catégories, il n'en est plus de même. Nous pouvons sans doute juxtaposer les mots ce étourdi est vert; plus intense est rond; cette maison est égale; nous pouvons, dans un énoncé relationnel de la forme $a$ est semblable à $b$, substituer cheval à semblable, mais nous n'obtenons toujours ainsi qu'une série de mots, où chaque mot comme tel a bien un sens, ou renvoie à un ensemble complet de sens, mais en principe nous n'obtenons pas une unité de sens se suffisant à elle-même ${ }^{2}$.

À titre d'illustration des lois de la morphologie pure, Husserl énonce, dans le $\S 13$, quelques principes de composition des significations d'après leur appartenance catégorielle ${ }^{3}$. Ainsi, « à une signification nominale quelconque $S$ et à une quelconque signification adjective $p$, correspond la forme primitive $S p$ (par exemple maison rouge); le résultat est, en vertu d'une loi, une nouvelle signification de la catégorie "signification nominale" $\gg{ }^{4}$. Autres exemples: la mise en relation de deux matières nominales (la pomme, l'arbre) par la conjonction « et » est une authentique unité de signification et c'est elle-même une matière nominale; la mise en relation de deux

\footnotetext{
${ }^{1}$ Ibid., $\S 10$, p. 112-113.

${ }^{2}$ Ibid., $§ 10$, p. 113-114.

${ }^{3}$ Ibid., $\S 13$, p. 125.

${ }^{4}$ Ibid., $§ 13$, p. 125-126.
} 
propositions (Il pleut, L'herbe est verte) par la conjonction «et» est une authentique unité de signification et est une proposition; par contre, la mise en relation d'une proposition et d'une matière nominale (Il pleut, la pomme) par la conjonction « et » est un non-sens.

\section{Bonne formation et signifiance}

Or, dans son article «Synctactical and semantical categories » de l'Encyclopedia of Philosophy, Yehoshua Bar-Hillel critique en des termes très durs la « superficialité » de ces analyses husserliennes:

[Husserl] soutenait que nous déterminons si deux expressions appartiennent ou non à la même catégorie de signification, ou si deux significations vont ou non ensemble, par une " évidence apodictique ». Mais ses exemples et sa terminologie - par exemple, l'utilisation de l'expression «matière adjective » — indiquent que son évidence apodictique n'était rien d'autre qu'une sorte d'intuition grammaticale non sophistiquée, qu'il hypostasia comme aperçu du royaume des significations ${ }^{1}$.

Dans cette dénonciation de l'hypostase de catégories qui ne seraient en fait que des déterminations linguistiques propres à telle ou telle langue ou à tel ou tel groupe de langues, on semble reconnaître un motif classique, typiquement adressé au rationalisme universaliste par les tenants du "linguistic turn », et qui a notamment inspiré la critique par Benveniste de la liste aristotélicienne des catégories ou encore la critique par Sapir et Whorf de toute prétention à dégager des universaux linguistiques et, à travers eux, des universaux logiques et ontologiques.

Mais l'article de Bar-Hillel contient aussi et surtout une critique plus spécifique, qui reproche à Husserl de s'en être tenu, au moment de dégager les catégories de la signification et les lois de leurs combinaisons signifiantes, à des considérations purement syntaxiques sans traiter des questions proprement sémantiques. Dans son article, en effet, Bar-Hillel opère la distinction entre le plan syntaxique et le plan sémantique - avec leurs catégories et règles de composition spécifiques - à partir de la distinction entre «bonne formation » et « signifiance » des complexions de significations :

${ }^{1}$ Y. Bar-Hillel, «Synctactical and semantical categories », in The Encyclopedia of Philosophy, MacMillan, London, New York, 1967, vol. 8, p. 58. 
Le fondement de toute théorie des catégories syntaxiques est le fait linguistique que dans tous les langages naturels il y a des séquences de (un ou plusieurs) mots qui sont mutuellement interchangeables dans tous les contextes bien formés salva beneformatione - c'est-à-dire que la bonneformation (la grammaticalité, la correction syntaxique) est préservée dans l'échange - et qu'il y a d'innombrables autres séquences qui ne se trouvent pas dans cette relation les unes avec les autres. Toute théorie des catégories sémantiques repose sur un fait similaire, en remplaçant cette fois «bien formé » par «signifiant (meaningful)» ou «sémantiquement correct», et «beneformatione » par « significatione ${ }^{1}$.

Ce sur quoi Bar-Hillel insiste ici, c'est qu'il y a en fait deux niveaux de nonsens, celui de la malformation syntaxique et celui de l'incongruité sémantique. Ainsi, poursuit Bar Hillel en reprenant un exemple donné par Noam Chomsky, "l'expression anglaise "Colorfull green ideas sleep furiously" est, au moins prima facie, syntaxiquement bien formée. Et pourtant elle est sémantiquement dépourvue de signification $»^{2}$. Aux règles de composition syntaxiques - qui autorisent par exemple les substitutions d'adjectif à adjectif — s'ajoutent en fait des règles de composition sémantiques - qui limitent les substitutions possibles d'adjectif à adjectif puisque n'importe quel adjectif ne peut sans incongruité se rapporter à n'importe quel nom. En plus donc de la séparation de ces deux types d'impossibilité que sont le non-sens et le contresens - séparation qui, reconnaît Bar-Hillel, est incontestablement un acquis important de la quatrième Recherche logique husserlienne ${ }^{3}$-, il faut, selon Bar-Hillel, maintenir, au sein du premier type, une séparation entre deux sortes différentes de non-sens.

En suivant Bar-Hillel dans cette mise au point, il faut bien reconnaître avec lui que ce sont essentiellement des catégories et lois de composition syntaxiques - autour des notions de «nom », d' "adjectif », de « relation » ou de "proposition» - que Husserl semble mettre en avant dans son analyse; et que c'est la bonne formation syntaxique plutôt que la cohérence

\footnotetext{
${ }^{1}$ Ibid., p. 57.

${ }^{2}$ Ibid.

${ }^{3}$ Bar-Hillel rend explicitement hommage à Husserl sur ce point : « Husserl mérite certainement beaucoup d'estime pour avoir distingué non-sens (Unsinn) et contresens (Widersinn), ou, en termes modernes, pour avoir distingué les séquences (strings) qui violent des règles de formation et les séquences qui peuvent être réfutées par des règles de déduction » (Ibid., p. 58).
} 
sémantique qui semble le préoccuper. Cette dernière est même, nous l'avons vu, très explicitement laissée de côté :

Quand on substitue librement les unes aux autres des matières à l'intérieur de leur catégorie, il peut en résulter des significations (des propositions entières ou des membres possibles de propositions) fausses, absurdes ou risibles, mais il doit nécessairement en résulter des significations unitaires ou des expressions grammaticales dont le sens peut être réalisé comme unité de sens ${ }^{1}$.

\section{Théorie des types logiques}

Que n'importe quelle matière nominale ne puisse pas se combiner avec n'importe quelle matière adjective ou relationnelle, ou, pour le dire autrement, que n'importe quel argument ne puisse pas saturer n'importe quelle fonction propositionnelle sans absurdité et même sans paradoxe, c'est par contre ce dont Russell et Frege ont (douloureusement) pris conscience dès 1902. Que, contrairement à l'universalisme logique préalablement présupposé, la grammaire pure logique mise au fondement de l'idéographie du calcul extensionnel, et qui reposait sur la distinction des concepts (éventuellement relationnels) et des objets, ou des fonctions propositionnelles (à une ou plusieurs places) et de leurs arguments, doive impérativement intégrer une théorie des types logiques sous peine de contradiction, c'est ce que, dans leur correspondance célèbre, Russell constate et Frege regrette ${ }^{2}$. De l'ensemble des fondateurs de Rome, on ne peut se demander s'il est luimême un fondateur de Rome. Un ensemble d'objets n'a pas - ne peut pas avoir - les mêmes propriétés que ses membres, et en particulier la propriété qui les rassemble en lui et le constitue en tant qu'ensemble. À l'inverse, les ensembles possèdent des propriétés que ne possèdent pas leurs membres ; l'ensemble des fondateurs de Rome a une cardinalité - 2 - que n'avaient pas Romulus et Remus ${ }^{3}$.

${ }^{1}$ E. Husserl, Recherches logiques, IV, op. cit., tome II, § 10, p. 113.

${ }^{2}$ G. Frege, Correspondance avec Bertrand Russell, trad. fr. C. Webern, Paris, E.P.E.L., 1994.

${ }^{3}$ B. Russell, «Les paradoxes de la logique », Revue de métaphysique et de morale vol. 14, 1906, p. 627-650 ; «Mathematical logic as based on the theory of types », American Journal of Mathematics, 1908, trad. fr. F. Rivenc et P. de Rouilhan (éds.), Logique et fondements des mathématiques, Paris, Payot, 1992. 
Dans cette perspective de la théorie des types, on peut alors expliquer, par exemple, que l'on ne peut attribuer une couleur à un objet abstrait tel qu'un nombre comme on le fait pour un objet matériel. Pour les logicistes, 2 est l'ensemble de toutes les paires - de tous les ensembles qui ont la cardinalité « $2 »-$; c'est donc un ensemble d'ensembles et donc un objet qui est de type logique nettement supérieur aux objets dont se composent les ensembles (les paires) dont 2 lui-même se compose. C'est pourquoi, tandis que Romulus et Remus peuvent avoir une couleur, ni l'ensemble des fondateurs de Rome, ni 2 en tant qu'ensemble formé de cet ensemble et des autres paires, ne peut avoir une couleur ${ }^{1}$.

$\mathrm{Si}$, comme Carnap en dessine le projet dans l'Aufbau, on parvient à reconstruire, niveau par niveau, tous les objets du discours scientifique comme des ensembles de relations entre des objets de niveau inférieur - les molécules comme des ensembles de relations entre atomes; les organites comme des ensembles de relations entre molécules; les cellules comme des ensembles de relations entre organites ; .. - on attribue aussi à chacun de ces objets un type logique bien défini, lequel explique alors qu'on ne puisse,

${ }^{1}$ «L'essence technique de la théorie des types, dit Russell, se réduit à ceci : étant donné une fonction propositionnelle $\varphi(x)$ dont toutes les valeurs sont vraies, il y a des expressions qu'il n'est pas légitime de substituer à x. Par exemple : toutes les valeurs de "si $x$ est un homme, $x$ est mortel" sont vraies, et non pouvons en inférer "si Socrate est un homme, Socrate est mortel", mais nous ne pouvons en inférer : "Si la loi de contradiction est un homme, la loi de contradiction est mortelle". La théorie des types déclare qu'il s'agit là d'un ensemble de mots dépourvus de sens, et donne des règles pour déterminer les valeurs que $x$ peut recevoir dans $\varphi(\mathrm{x})$. Le détail comporte des difficultés et des complications, mais le principe général n'est que la forme plus précise d'un principe depuis toujours reconnu. Dans la logique traditionnelle, on avait coutume de souligner qu'une expression comme "la vertu est triangulaire" n'est ni vraie ni fausse, mais aucune tentative n'était faite pour parvenir à un ensemble de règles permettant de décider si une série donnée de mots était ou non signifiante. C'est ce que réalise la théorie des types. Aussi ai-je par exemple affirmé plus haut que "les classes de choses n'étaient pas des choses". Ce qui veut dire : "Si "x est membre de la classe $\alpha$ " est une proposition et $\varphi x$ est une proposition, alors $\varphi \alpha$ n'est pas une proposition, mais une collection de symboles dépourvue de sens" " (B. Russell, Principles of mathematics (1903), London, Allen \& Unwin, 1964, introduction à la seconde édition (1937), p. XIV, trad. fr. dans Écrits de logique philosophique, Paris, P. U. F., p. 19). « Toute fonction propositionnelle $\varphi(\mathrm{x})$ a, en plus de son parcours de vérité (range of truth), un parcours de signifiance (range of significance), c'est-à-dire un parcours au sein duquel $\mathrm{x}$ doit se trouver si $\varphi(\mathrm{x})$ doit être une proposition, qu'elle soit vraie ou fausse » (ibid., appendice B, § 497, op. cit., p. 523, trad. fr. op. cit., p. 192). 
sans incongruité, attribuer à cet objet des propriétés qui relèvent d'un autre niveau logique ${ }^{1}$. Les classes sociales (objets spirituels) ne peuvent se voir attribuer les mêmes propriétés que les désirs (objets hétéropsychiques) que les atomes (objets physiques) ou que les sensations (objets autopsychiques). La bourgeoisie n'est pas perverse comme peut l'être un désir sexuel ; elle n'a pas une valence de 2 comme peut l'avoir un atome et n'est pas plus ou moins vive comme peut l'être une sensation. La théorie des types logiques, on le voit, complète la grammaire pure de l'idéographie - avec ses catégories de fonctions propositionnelles et d'arguments et leurs lois de combinaison, c'est-à-dire de la saturation des premières par les seconds - par une série de règles relatives aux catégories sémantiques et à leurs propres lois de combinaison $^{2}$.

\section{Erreurs de catégorie}

Que les contraintes grammaticales sous-jacentes à la rationalité scientifique et quotidienne ne se réduisent pas à celles que mettent en avant l'idéographie et la théorie des types logiques, c'est, on le sait, ce que va mettre en avant une seconde génération de philosophes analytiques, désormais soucieux d'analyse du langage ordinaire et non seulement d'analyse logique. En particulier, Gilbert Ryle thématisera sous l'expression d'" erreurs de catégorie » les confusions quant à la catégorie sémantique dont relève une expression et, par conséquent, les combinaisons dans lesquelles elle peut

1 R. Carnap, La construction logique du monde (1928), trad. fr. T. Rivain et E. Schwartz, Paris, Vrin, 2002. L’ouvrage s'ouvre par une citation de Russell : « La maxime suprême de la philosophie scientifique est la suivante : partout où c'est possible, des constructions logiques doivent être substituées aux entités inférées » (p. 57).

${ }^{2}$ Dans la Construction logique $d u$ monde, la théorie russellienne des types logiques est réinterprétée en une théorie plus générale des "sphères d'objets », conçues comme catégories de signification ( op . cit., § 29, p. 93-94). La cinquième et dernière partie de l'ouvrage, intitulée « Clarification de certains problèmes philosophiques sur la base de la théorie de la construction », est consacrée à disqualifier comme nonscientifiques des questions qui ne peuvent recevoir une traduction claire dans les termes du système de la science mis en évidence dans les chapitres précédents, en raison notamment « de la négligence de distinctions dans les types logiques de différentes sortes de concepts » (R. Carnap, « Intellectual autobibliography », in The philosophy of Rudolf Carnap, The library of living philosophers, London, Cambridge University Press, 1963, p. 45). 
entrer sans incongruité. Depuis son célèbre «Systematically misleading expressions " - où Ryle avait traqué une multitude d'énoncés syntaxiquement bien formés mais sémantiquement fourvoyants ${ }^{1}$ - jusqu'à son texte «Categories » - où il avait interprété la théorie aristotélicienne des catégories comme visant à identifier et dégager une variété de «formes propositionnelles » distinctes sous une communauté de structure syntaxique (la prédication $)^{2}$ - , Ryle n'a cessé de chercher à penser les contraintes sémantiques et non seulement syntaxiques qui pèsent sur les combinaisons sensées d'expressions.

Et lorsque, dans The concept of mind, il reprochera au " mentalisme » et singulièrement à Descartes, d'avoir fait de l'esprit une substance distincte du corps mais analogue à lui, et d'avoir aussi conçu, les objets et états mentaux sur le modèle des objets et états physiques, c'est encore une confusion sémantique qu'il dénonce :

J'espère prouver que [le dogme du «fantôme dans la machine»] est entièrement faux, et faux non pas dans le détail mais dans le principe même. Ce n'est pas simplement l'assemblage d'erreurs singulières. C'est une seule grosse erreur et une erreur d'un type particulier, à savoir que c'est une erreur de catégorie. Il [le dogme] représente les faits de la vie mentale comme s'ils appartenaient à un type ou à une catégorie logique alors qu'ils appartiennent en fait à une autre ${ }^{3}$.

Deux pages plus loin, Ryle précise la nature de cette grave méprise qui est à l'origine de toute une conception erronée du mental :

Mon objectif déconstructif est de montrer qu'une famille d'erreurs de catégories radicales est la source de la théorie de la double vie. La représentation d'une personne comme un fantôme mystérieusement installé dans une machine provient de l'argument suivant. Puisque les pensées, les sentiments et les actions intentionnelles d'une personne ne peuvent être décrits seulement dans les termes de la physique, de la chimie et de la physiologie, ils doivent être décrits dans des langages homologues (counterpart idioms). Puisque le corps humain est une unité organisée complexe, l'esprit humain doit être une autre unité organisée complexe, bien que faite d'une sorte différente de substance et d'une sorte différente de

${ }^{1}$ G. Ryle, «Systematically misleading expressions » (1932), Proceedings of the Aristotelian Society, 1932, vol. XXXII, p. 139-170.

2 G. Ryle, "Categories", Proceedings of the Aristotelian Society, 1938, vol. XXXVIII, p. 189-206, réédité dans les Collected papers, London, Hutchinson, vol. 2. ${ }^{3}$ G. Ryle, The concept of mind, London, Hutchinson, 1949, p. 16. 
structure. Ou encore, puisque le corps humain, comme toute parcelle de matière, est un champ de causes et d'effets, l'esprit doit être un autre champ de causes et d'effets, bien que (Dieu soit loué) pas de causes et d'effets mécaniques ${ }^{1}$.

L'erreur de catégorie que Ryle dénonce ici, Lucie Antoniol ${ }^{2}$ l'interprète explicitement comme une confusion de catégories sémantiques :

On connaît, écrit Lucie Antoniol, les réactions « allergiques » de Ryle à toute espèce de naming theory of meaning. Or l'erreur des cartésiens consiste précisément à considérer les prédicats mentaux comme des noms et à postuler des lieux et des processus fantomatiques que ces noms puissent désigner. Selon Ryle, le « discours mental » n'a pas pour fonction de nommer quoi que ce soit, mais bien de qualifier les actions et réactions des gens (actions et réactions qui peuvent aussi bien être publiques que privées) $)^{3}$.

Pour Ryle, le problème «catégoriel » semble tout entier résider dans une confusion quant au type de lien qui existe entre certaines expressions du langage et les réalités qu'elles signifient. Plutôt que de dénommer des entités ou événements spécifiques, les termes mentaux serviraient seulement à qualifier les entités et événements désignés par les termes "physiques ». S'appuyant sur un article de Th. W. Bestor, Antoniol formule cette interprétation de manière limpide :

Ryle veut prouver que nos prédicats mentaux ont une fonction logique «adverbiale» plutôt que «substantive» [...] Ils ne sont pas les noms d'habitants d'un monde spécial. Ils sont les accompagnateurs spéciaux de nos explications des actions et passions ordinaires. Ils nous disent que certaines affaires humaines sont menées ou subies dans certaines dispositions, conduites avec un certain style, d'une certaine manière, dirigées par des personnes prêtes à faire ou subir d'autres choses, ou bien visant un certain résultat ${ }^{4}$.

On sait que, dans ses Recherches philosophiques puis plus longuement encore dans ses Remarques sur la philosophie de la psychologie, Wittgenstein énoncera des thèses très similaires. La conception « mentaliste » de la psychologie repose, selon Wittgenstein, sur une interprétation simpliste

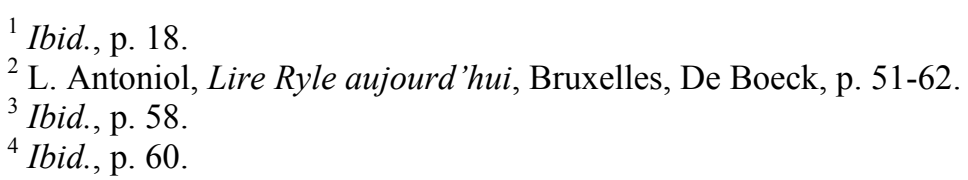


(augustinienne) des substantifs psychologiques (douleur, croyance, désir, espoir, etc.) comme désignant nécessairement des objets; et une interprétation simpliste (augustinienne) des énoncés psychologiques ( $« \mathrm{je}$ ressens une douleur à la jambe », «j'éprouve de l'amour pour...», «je souhaite que...», etc.) comme des énoncés nécessairement descriptifs. Et puisque ces «objets désignés » et ces « faits décrits » ne sont pas des objets et des faits " extérieurs », on suppose que ce sont des objets et faits d'un autre genre qui existent ou se déroulent parallèlement aux objets et faits « extérieurs ».

Cette critique radicale du mentalisme a parfois mené les commentateurs à rapprocher Wittgenstein du behaviorisme qui conteste l'existence des états mentaux et propose de s'en tenir à l'étude de ce qui est intersubjectivement observable, à savoir les comportements. Wittgenstein se défend pourtant lui-même d'une telle position. Le combat de Wittgenstein est « grammatical ${ }^{1}$ - s'il y a behaviorisme, c'est un behaviorisme logique, qui s'intéresse au sens des concepts psychologiques et à leurs conditions de satisfaction comportementales - et sa cible est l'image - suggérée par une mauvaise interprétation du langage - selon laquelle la psychologie aurait pour objet des réalités mentales à découvrir «sous la surface» des comportements. Wittgenstein ne dit pas pour autant que la psychologie doit s'en tenir à étudier la surface, ou qu'il n'y a rien sous la surface. Wittgenstein conteste plutôt les images mêmes de surface et de profondeur ou d'intérieur et d'extérieur :

C'est se fourvoyer exactement de la même manière que de dire qu'il n'y a que surface et rien dessous et de dire qu'il y a quelque chose sous la surface et non pas seulement la surface ${ }^{2}$.

Le défaut commun au mentalisme et au behaviorisme est de concevoir le jeu de langage psychologique sur le modèle augustinien. En introduisant dans ce jeu des formules unificatrices telles qu' «état mental », « objet privé » ou "phénomène interne », le mentalisme l'a rendu plus mystérieux qu'il n'était; il a rangé les termes psychologiques « dans le mauvais tiroir $»^{3}$.

${ }^{1}$ L. Wittgenstein, Recherches philosophiques (1933-1949), Paris, Gallimard, 2004, $\S 307$, p. 153.

${ }^{2}$ L. Wittgenstein, Notes sur l'expérience privée (1934-1936), Mauvezin, T.E.R., 1982, p. 51.

${ }^{3}$ L. Wittgenstein, Remarques sur la philosophie de la psychologie (1947-1948), I, Mauvezin, T.E.R, 1989, $\S 380$, p. 82. 


\section{Multiplicité des catégories sémantiques}

Plus généralement, le second Wittgenstein n'a eu de cesse de dénoncer les confusions qui résultent du fait de sous-estimer les variations sémantiques sous une même forme syntaxique: "Nous n'avons pas conscience de l'indicible disparité existant entre les jeux de langage quotidiens, parce que les vêtements de notre langage uniformisent tout ${ }^{1}$. Contrairement à ce que soutenait le Tractatus ${ }^{2}$, pour lequel le langage avait pour seule fonction de décrire comment est le monde - de dire qu' " il en est ainsi », c'est-à-dire d'énoncer des états de choses —, il existe, disent les Recherches philosophiques, une multiplicité indéfinie de sortes de phrases $^{3}$; toutes n'ont pas le même statut parce que toutes n'ont pas le même usage. Certes, le langage tout entier a "résonnance propositionnelle (Satzklang) » ${ }^{4}$, mais en fait «le schéma sujet-prédicat sert de projection pour un nombre infini de formes logiques différentes $»^{5}$. Le langage est un poste d'aiguillage : ses propositions sont autant de manettes dont l'apparence globalement similaire - du fait qu'elles doivent tenir en main, être manipulées - cache en fait des fonctions différentes ${ }^{6}$. C'est pourquoi il faut aller au-delà de la " grammaire de surface », qui se laisse leurrer par les analogies linguistiques ${ }^{7}$, pour parvenir à une "grammaire profonde ${ }^{8}$ susceptible de fonder une authentique analyse logique.

À qui ignore l'usage qui en est fait dans les divers jeux de langage, tous les mots se ressemblent, comme se ressemblent encore les différentes lignes tracées sur une carte d'état-major pour qui ne sait rien de leurs

${ }^{1}$ L. Wittgenstein, Recherches philosophiques (1933-1949), op. cit., partie II, p. 314.

2 L. Wittgenstein, Tractatus logico-philosophicus (1921), Paris, Gallimard, 1993, $\S 4.5$, p. 70 : «La forme générale de la proposition est : ce qui a lieu est ainsi et ainsi ».

${ }^{3}$ L. Wittgenstein, Recherches philosophiques (1933-1949), op. cit., § 23, p. 125.

${ }^{4}$ Ibid.., $§ 134$, p. 170. Cf. aussi Remarques philosophiques (1930), Paris, Gallimard, $1975, \S 85$, p. 109.

${ }^{5}$ L. Wittgenstein, Grammaire philosophique (1930-1933), Paris, Gallimard, 1980, App. 2, p. 211

${ }^{6}$ L. Wittgenstein, Recherches philosophiques (1933-1949), op. cit., § 12, p. 33. Cf. aussi Grammaire philosophique (1930-1933), op. cit., § 20, p. 66-67.

7 «Les classifications que font philosophes et psychologues sont comme si on voulait classer les nuages selon leur forme"» (L. Wittgenstein, Remarques philosophiques (1930), op. cit., § 154, p. 173).

${ }^{8}$ L. Wittgenstein, Recherches philosophiques (1933-1949), op. cit., § 664, p. 237.

\section{1}


fonctions ${ }^{1}$. L'analyse pragmatique, par contre, fait apparaître différents types de mots, régis par des règles d'usage très différentes ${ }^{2}$. Et la classification de ces différents types doit aller bien au-delà de la catégorisation «substantifs, adjectifs, verbes, adverbes, ...», puisqu'aux contraintes syntagmatiques s'ajoutent des impossibilités d'intersubstitution que les linguistes appellent «paradigmatiques » :

Si on nous remontre que le langage peut tout exprimer à l'aide de substantifs, adjectifs et verbes, il nous faut dire qu'il est alors nécessaire, dans chaque cas, de distinguer entre des types tout à fait différents de substantifs, etc., étant donné que des règles grammaticales différentes sont valables pour eux. Ce qui le montre, c'est qu'il n'est pas permis de les substituer l'un à l'autre. Par là se montre que leur caractère de substantifs était seulement du superficiel et que nous avons affaire en réalité à des alliances de mots tout à fait différentes. Les alliances du mot ne sont déterminées que par toutes les règles grammaticales qui régissent un mot et, considéré ainsi, notre langage a une masse de types de mots différents ${ }^{3}$.

La forme sous laquelle une «signification » est projetée dans le langage substantif, verbe, adjectif, proposition complète, ... - ne nous dit en fait pas encore quel est son statut, ni quel rapport elle entretient avec le signe linguistique qui la représente. Ainsi, la dénomination - le fait pour un nom d'être " attaché comme une étiquette à une chose » ${ }^{4}$ - n'est qu'un des jeux de langage, qu'une des fonctions possibles du nom ${ }^{5}$. Tous les termes que nous appelons « noms » ne désignent pas un objet de la même manière.

En disant : « Chaque mot du langage désigne quelque chose », nous n'avons encore absolument rien dit, à moins que nous n'ayons expliqué de façon précise quelle distinction nous souhaitons faire ${ }^{6}$.

\footnotetext{
${ }^{1}$ Ibid., § 21, p. 67.
}

${ }^{2}$ Bien qu'ils s'alignent les uns à côté des autres comme les outils sur un établi de menuisier, les mots qui composent ces phrases sont destinés à des usages aussi divers que les outils du menuisier (Ibid., § 31, p. 75).

${ }^{3}$ L. Wittgenstein, Remarques philosophiques (1930), op. cit., § 92, p. 113-114.

${ }^{4}$ L. Wittgenstein, Recherches philosophiques (1933-1949), op. cit., § 15, p. 34; § 26, p. 41.

${ }_{5}^{5}$ L. Wittgenstein, Remarques sur la philosophie de la psychologie (1947-1948), I, op. cit., § 614, p. 137. Cf. aussi Recherches philosophiques (1933-1949), op. cit., $\S 27$, p. 41 .

${ }^{6}$ L. Wittgenstein, Recherches philosophiques (1933-1949), op. cit., § 13, p. 33.

\section{2}


$\mathrm{Au}$ fond, c'est presque à tort que nous mettons en avant la notion générale de «nom» :

Nous appelons «nom » des choses très diverses; le mot «nom » caractérise de nombreux types d'emplois différents d'un mot qui sont apparentés les uns aux autres de bien des manières différentes ${ }^{1}$.

$\mathrm{Si}$, pour le second Wittgenstein, l'analyse idéographique frégéo-russellienne doit faire place à l'analyse du langage ordinaire, c'est donc en fait parce qu'il y a une multiplicité irréductible de jeux de langage, entre lesquels n'existent que des analogies partielles ${ }^{2}$, des « ressemblances de famille (Familienähnlichkeiten) $»^{3}$, et non une structure idéale commune.

Pour les philosophes du langage ordinaire, la théorie des types logiques ne suffit évidemment pas à rendre compte de cette multiplicité irréductible des jeux de langage. Le projet logiciste défendu dans l'Aufbau consistait en fait à les concevoir comme autant de niveaux d'une structure commune, mais cela suppose donc que tous les objets du discours puissent effectivement être (re)construits par des relations purement logiques (ensemblistes) les uns à partir des autres, ce que Wittgenstein et Ryle estiment tout à fait illusoire. Dans Categories, Ryle affirme explicitement que certaines violations des règles sémantiques ne relèvent pas des problèmes logiques de paradoxes ou de cercles vicieux auxquels Russell s'est intéressé $^{4}$. À Aristote et à Kant, Ryle adresse le reproche de présupposer qu' « il y a un catalogue fini de catégories ou de types ${ }^{5}$. Certes, la hiérarchie des types logiques a l'avantage d'être potentiellement infinie, mais elle limite singulièrement le type de rapport que les catégories peuvent entretenir les unes avec les autres. Or, pour Ryle, la liste des types n'est pas et ne pourra jamais être complète ni même capturée par quelques règles de formation

${ }^{1}$ Ibid., § 38, p. 48. Cf. aussi Remarques philosophiques (1930), op. cit., § 92, p. 114 : " Si on donne un nom à un corps, on ne peut pas donner un nom dans le même sens à sa couleur, sa forme, sa situation, sa surface. Et inversement». Cela ne peut que conduire à un emploi très « embrouillé » du mot « objet » (Ibid., premier appendice, p. 289).

2 «Un jeu de langage analogue à un fragment d'un autre» (L. Wittgenstein, Remarques sur la philosophie de la psychologie (1947-1948), I, op. cit., § 936, p. 196).

${ }^{3}$ Sur la notion d' « air de famille », voir en particulier L. Wittgenstein, Recherches philosophiques (1933-1949), op. cit., §§ 66-67, p. 64-65.

${ }^{4}$ Ibid., p. 180.

${ }^{5}$ Ibid., p. 179. 
prédéfinies, raison pour laquelle le projet idéographique est sans doute voué à l'échec :

Je ne pense pas qu'on puisse jamais dire d'un langage symbolique donné en logique formelle que ses symboles sont maintenant adéquats pour la symbolisation de toutes les différences possibles de type ou de forme ${ }^{1}$.

Dans la conférence inaugurale des Tarner Lectures - prononcées en 1953 et publiées l'année suivante sous le titre «Dilemmas» —, Ryle écrira dans la même veine :

Certains aristotéliciens loyaux, qui, comme tous les loyalistes ont ossifié l'enseignement du maître, ont traité sa liste des catégories comme si elle fournissait les cases dans lesquelles pourrait ou devrait être rangé chaque terme utilisé ou utilisable dans le discours technique et non-technique. Chaque concept doit être soit de la Catégorie I, soit de la Catégorie II, ... soit de la Catégorie X. Mais de nos jours il existe des penseurs qui, loin de trouver cette réserve de cases intolérablement exiguë, la trouvent trop généreuse; et qui sont prêts à dire de n'importe quel concept qu'on leur présente « Est-ce une Qualité ? Si non, c'est une Relation. » [...] La vérité, c'est qu'il n’y pas tout juste deux ou tout juste dix métiers logiques différents qui sont ouverts aux termes et concepts que nous employons dans le discours ordinaire et technique ; il y a un nombre indéfini de tels métiers et un nombre indéfini de dimensions à leurs différences ${ }^{2}$.

\section{Grammaires catégorielles}

Cette artificialité de la théorie des types logiques et son insuffisance à rendre compte de la multiplicité indéfinie des catégories sémantiques ${ }^{3}$, c'est d'ailleurs ce que, avant les philosophes du langage ordinaire, avait également pointé Stanisław Leśniewski. Et il est évidemment très intéressant de constater qu'il s'était précisément tourné vers la morphologie pure husserlienne pour élaborer une théorie des catégories sémantiques concur-

${ }^{1}$ Ibid., p. 179.

${ }^{2}$ G. Ryle, «Dilemmas », in Dilemmas, Cambridge, Cambridge University Press, 1954, p. 10.

${ }^{3}$ E. Husserl, Recherches logiques, IV, op. cit., tome II, §§ 4-9. Jocelyn Benoist souligne l'influence, à cet égard, de la conception de la « syntaxe » développée par Anton Marty (Phénoménologie, sémantique, ontologie, Husserl et la tradition logique autrichienne, Paris, P.U.F., 1997, p. 105). 
rente de la hiérarchie russellienne des types logiques qui lui semblait artificielle et dénuée de fondement intuitif. Explicitement inspirée de la quatrième Recherche logique, la grammaire de Leśniewski met en œuvre deux catégories de base (phrases et noms) et permet de construire, à partir d'elles, une multitude de catégories dérivées, comme celle des expressions qui, jointes à un nom, forment avec une lui une phrase (les prédicats), celle des expressions qui, jointes à un prédicat, forment avec lui un prédicat (les adverbes) ou celle des expressions qui, jointes à un nom, forment avec lui un autre nom (les épithètes).

Ce principe de construction s'appuie en fait sur la distinction husserlienne des catégorèmes et des syncatégorèmes, mais sa force est évidemment de définir précisément la forme de chaque syncatégorème comme une certaine "fonction" des deux catégories de base. De plus, comme ces catégories dérivées permettent à leur tour de construire de nouvelles catégories dérivées, on a là un principe formel de caractérisation d'un nombre potentiellement infini de catégories, susceptible donc de rendre compte de l'ensemble des formes d'expression du langage logique, y compris de nouvelles formes d'expression qui apparaissent au cours du développement du système logique.

Comme l'indiquera Leśniewski lui-même quelques années plus tard, cette théorie des catégories sémantiques est en fait équivalente à la théorie des types logiques sur le plan de ses conséquences formelles - elle distingue par exemple nettement la conjonction propositionnelle ( $« \mathrm{Il}$ a 4 frères et 3 sœurs ») de la conjonction nominale (« Il a 4 ans et 3 mois ») -, mais « elle se rapproche plutôt, par son aspect intuitif, de le tradition des " catégories » d'Aristote, des «parties du discours » de la grammaire traditionnelle et des « catégories de signification » d'Edmund Husserl ${ }^{1}$. Et, par « intuitif», ce que Leśniewski veut dire, ce n'est pas seulement que sa morphologie pure est plus proche des langues naturelles que l'idéographie frégéo-russellienne, mais aussi et surtout qu'elle ne se contente pas de mettre au point des règles de bonne formation des expressions du langage logique, mais qu'elle s'efforce en même temps de rendre compte des lois de composition des significations elles-mêmes; comme chez Husserl, les catégories mises en œuvre et les lois de composition qui les régissent sont directement liées à la

1 S. Leśniewski, «Gründzüge eines neuen Systems der Grundlagen der Mathematik », 1929, Fundamenta Mathematicae, trad. angl. dans Collected works, Dordrecht, Kluwer et Warszawa, Polish Scientific Publisher, 1992, p. 424.

185 
possibilité même de produire du sens (make sense), raison pour laquelle il s'agit, selon Leśniewski, de catégories sémantiques autant que syntaxiques ${ }^{1}$.

En 1935, un disciple de Leśniewski, Kazimierz Ajdukiewicz, développera la théorie de son maître en la dotant d'un langage formel d'index fractionnaires (avec, par exemple, la forme « $\mathrm{n} / \mathrm{s} \backslash \mathrm{n} »$ pour une expression comme un verbe transitif qui, mise entre deux noms, forme une phrase (sentence)) et en la munissant d'algorithmes de résolution qui permettent de vérifier mécaniquement la grammaticalité de séquences de mots :

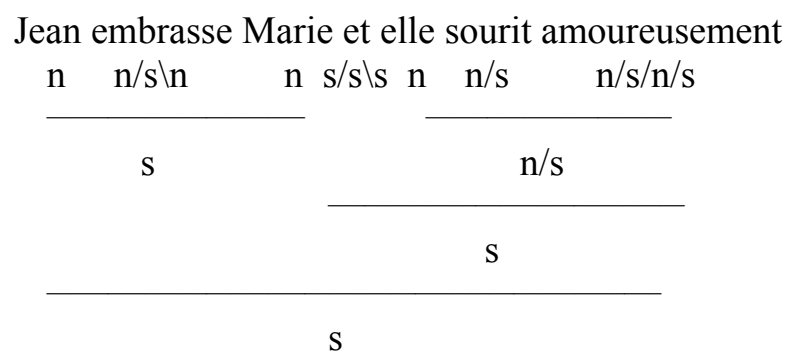

Comme Leśniewski, Ajdukiewicz insiste sur la capacité qu'a son système de prouver, de façon plus élégante et plus simple que la théorie des types, la mauvaise formation d'énoncés comme ceux des paradoxes logiques. On parle bien ici de «mauvaise formation » car, à l'instar de la théorie des types logiques qui, en déployant la forme logique exacte (et souvent très complexe) de tous les objets de type supérieur, fait apparaître, dans leur expression symbolique même, l'illégitimité de leur attribuer des propriétés de niveaux inférieurs - une fois entièrement déployé, l'énoncé apparaît comme syntaxiquement mal formé - , les grammaires catégorielles font apparaître comme «mal formées» des expressions qui respectent pourtant la syntaxe élémentaire de la prédication d'une «forme adjective» à une «forme nominale» :

« Cet arbre est vert » est de catégorie $\mathrm{S}$

Une fois «nominalisée » pour devenir le sujet d'un nouvel énoncé ${ }^{1}$, cette expression doit conserver quelque chose de sa catégorie grammaticale

${ }^{1}$ Dans Un développement des systèmes de Stanislaw Leśniewski, Protothétique Ontologie - Mériologie, Berne, P. Lang, 1984, p. 155-156, Denis Miéville recommande l'expression de « catégories sémantico-syntaxiques».

186 
initiale, et dès lors ne se voir attribuer que des prédicats qui conviennent à des phrases :

«Que cet arbre est vert est plaisant » est grammaticalement correct «Que cet arbre est vert est vert» ne l'est pas

Cependant, même si la catégorisation permise par les grammaires catégorielles est nettement plus souple que celle des théories des types logiques, on voit bien qu'elle ne peut rendre compte de la multiplicité des catégories sémantiques que revendiquent les philosophes du langage ordinaire. C'est pourquoi Yehoshua Bar-Hillel adresse à Leśniewski le même reproche qu'à Husserl :

Le terme de Leśniewski « catégories sémantiques » doit être regardé comme un terme mal approprié (misnomer), puisque la catégorisation était fondée sur des considérations purement syntaxiques ${ }^{2}$.

Et de la même façon, à Ajdukiewicz, qui dit d'ailleurs lui-même théoriser «Die Synktaktische Konnexität» ${ }^{3}$, Béatrice Godard-Wendling reprochera de ne pas imposer de contrainte proprement sémantique sur le principe d'intersubstituabilité permettant d'identifier les «catégories de signification $»^{4}$ :

Recourant au test de la substitution pour répartir les différentes expressions du langage dans diverses classes catégorielles, Ajdukiewicz, qui considère pour-

${ }^{1}$ Là encore, les grammaires catégorielles ne font que développer ce que Husserl avait déjà thématisé dans la quatrième Recherche logique. Outre l'identification des types fondamentaux de significations et des lois essentielles de leur combinaison, disait Husserl, la «grammaire pure» doit dégager les «lois essentielles de modification » qui permettent à une signification d'une catégorie particulière de tenir, sous certaines conditions, la fonction d'une autre catégorie. Husserl donne l'exemple d'un moment de la forme qui, en vertu d'une modification de la signification, devient une matière nominale : dans « "si" est une particule», «si » tient la fonction d'un nom. De même, et moyennant de tout autres modifications, des matières adjectives ou des propositions entières peuvent être "nominalisées » (E. Husserl, Recherches logiques,IV, § 11, p. 115 à 119). Sur ce point, voir l'article de V. Cibotaru dans le même volume.

${ }^{2}$ Y. Bar-Hillel, « Synctactical and semantical categories », op. cit., p. 58.

${ }^{3}$ K. Ajdukiewicz, «Die Syntaktische Konnexität», Studia Philosophica, 1934, vol. 1, p. 1-27.

${ }^{4}$ Ajdukiewicz continue d'utiliser le terme husserlien de «Bedeutungskategorie ». 
tant que « la catégorie d'un mot est déterminée par sa signification », ne retient paradoxalement pas ce critère lors de l'affectation des catégories aux expressions, puisqu'il ne pose aucune contrainte sémantique sur la notion d'interchangeabilité. Il en résulte, par exemple, que les expressions « brille», « siffle» et «danse» se voient assigner la même catégorie, puisqu'en l'absence de contrainte sémantique, elles deviennent interchangeables dans le contexte «le soleil brille». La syntaxe d'Ajdukiewicz ne détecte en effet comme «non-phrases» que les amas de mots tels que «peut-être cheval si sera cependant brille », mais accepte comme étant des phrases des séquences sémantiquement déviantes comme "le soleil danse ». De cette absence de contrainte sémantique sur le mécanisme même de répartition des expressions dans diverses catégories, il s'ensuit que toutes les catégories de foncteur utilisées par cette syntaxe pour décrire les langues naturelles finissent par recouvrir ce qui était dénommé par les parties du discours. Ainsi la catégorie de foncteur $\mathrm{s} / \mathrm{n}$ correspond à la classe des verbes (intransitifs), la catégorie $\mathrm{n} / \mathrm{n}$ rend compte de tous les articles, etc. ${ }^{1}$.

\section{Contresens matériel}

Qu'en est-il alors de Husserl ? Husserl a très pertinemment distingué nonsens et contresens. En outre, au sein de ce dernier, il a à juste titre séparé le contresens formel - la contradiction logique telle que « Ce carré n'est pas carré »— du contresens matériel — tel que «Ce carré est rond $»^{2}$. Mais tout

${ }^{1}$ B. Godard-Wendling, "Les trois premières grammaires catégorielles", in Les grammaires catégorielles, Langages, déc. 2002, vol. 148, p. 53.

${ }^{2}$ Le paragraphe 11 de la troisième Recherche logique distinguait déjà les nécessités analytiques - qu'elles soient «pures» telles que «Un tout ne peut exister sans parties » ou « Si un certain $\alpha$ est dans une certaine relation avec un certain $\beta$, ce même $\beta$ est dans une certaine relation correspondante avec cet $\alpha$ » (E. Husserl, Recherches logiques, III, op. cit., t. II, § 11, p. 38) ou qu'il s'agisse de « particularisations » de ces lois pures, comme «Une maison ne peut exister sans son toit, ses murs et ses autres parties » ou «Il n'y a pas de père sans enfant »- des nécessités synthétiques a priori, comme «Une couleur ne peut exister sans une surface qui ait cette couleur ». Héritier de la notion bolzanienne d'analyticité au sens restreint, Husserl qualifie d'analytique l'a priori qui se caractérise par sa formalisation universelle. Les propositions analytiquement nécessaires «comportent une vérité pleinement indépendante de la nature concrète particulière de leurs objectités »; et, même dans celles qui contiennent des concepts matériels, « il doit être possible de remplacer chaque matière concrète, en maintenant intégralement la forme logique de la proposition, par la forme vide quelque chose, et d'éliminer toute position d'existence en passant à la forme de jugement correspondante ayant une généralité 
ce qui a été dit ici ne laisse-t-il pas penser qu'il convenait d'opérer une distinction similaire entre deux types de non-sens et reconnaître qu'à côté des non-sens formels - les malformations syntaxiques comme « Ce nombre est mange » - il y a aussi des non-sens matériels - les incongruités sémantiques comme «Ce nombre est bleu »? À la grammaire pure logique, n'aurait-il pas fallu ajouter une "grammaire matérielle » susceptible de rendre compte d'erreurs de catégorie qui dépassent la malformation syntaxique?

Il se fait qu'on trouve dans le $\S 89$ de Logique formelle et logique transcendantale une nette prise en considération de cette question de l'incongruité sémantique qui avait été écartée dans la quatrième Recherche logique. Pour être liés dans une signification unitaire, écrit Husserl, les éléments d'un jugement doivent « avoir affaire les uns aux autres ${ }^{1}$. Ainsi, la phrase «Cette couleur +1 donne 3 » n'a " pas de sens à proprement parler. [...] Les éléments isolés de la proposition ne sont pas dépourvus de sens, ce sont d'honnêtes sens, mais le tout n'offre pas un sens ayant une unité

inconditionnée ou le caractère d'une loi » (Ibid., § 12, p. 39). Une loi synthétique a priori, quant à elle, se caractérise par l'impossibilité d'une mise en variable illimitée (Bolzano) (Idées I, op. cit., §16, p. 56) et «inclut des concepts concrets d'une manière qui ne souffre pas salva veritate une formalisation de ces concepts » (Ibid., $\S 12$, p. 40). À l'a priori formel, Husserl oppose donc des a priori matériels, c'est-àdire des lois dont la nécessité a priori ne repose pas sur une mise en variables inconditionnée, mais bien restreinte à un genre d'objets, à une "région de l'être ". Ainsi, la loi « Il ne peut y avoir de couleur sans surface» ne repose pas sur la notion d'objet absolument quelconque, mais sur celui d'objet spatial. De même, les lois de la géométrie ne valent que pour les objets spatiaux et non pour les objets en général. Lorsque, dans le $\S 14$ de la quatrième Recherche logique, Husserl revenait sur la distinction de l'a priori formel et de l'a priori matériel, il opposait alors deux types de contresens : "le contresens formel ou analytique, par quoi nous comprenons précisément toute incompatibilité objective purement formelle, c'est-à-dire fondée dans l'essence pure des catégories de signification, indépendamment de toute "matière de la connaissance" concrète » et «le contresens matériel (synthétique) dont des concepts matériels (les ultimes noyaux matériels de signification) doivent répondre $»$. Le principe de non-contradiction, celui de la double négation ou du modus ponens sont «des lois garantissant contre le contresens formel » (Recherches logiques, IV, op. cit., t. II, $\S 14$, p. 130-131). Les propositions de la géométrie préservent par contre de contre-sens matériels tels que «un carré est rond» (Ibid., $\S 14$, p. 130).

${ }^{1}$ E. Husserl, Logique formelle et logique transcendantale, Paris, P.U.F., Epiméthée, 1957, § 89b, p. 294. 
harmonique; ce n'est pas un tout qui soit lui-même sens ${ }^{1}$. Mais, dit Husserl, cela veut donc dire que « la possibilité de la véritable effectuation de la possibilité d'un jugement (en tant qu'opinion) prend racine non seulement dans les formes syntaxiques mais aussi dans les matériaux syntaxiques $»^{2}$. Aux contraintes formelles de la bonne formation s'ajoutent les contraintes matérielles de la signifiance.

Cette distinction de la « forme » et des « matériaux » du jugement est sans doute la clé de l'explication de la mise à l'écart du problème de la compositionalité sémantique en 1901. Dans la mesure, en effet, où ce problème fait intervenir les "matériaux» du jugement, il ne concerne pas directement la logique formelle, qui était l'objet des Recherches logiques. Le $\S 89$ de Logique formelle et logique transcendantale confirme d'ailleurs explicitement cette explication :

Ce dernier fait [l'intervention des matériaux syntaxiques dans la signifiance], le logicien engagé dans la logique formelle le négligera facilement, du fait que son intérêt est dirigé d'une manière unilatérale vers le syntaxique — dont la multiplicité des formes appartient exclusivement à la théorie logique - et du fait qu'il algébrise les noyaux ${ }^{3}$.

Mais, s'il laisse cette question de côté dans sa logique formelle, Husserl la reprend-il ailleurs ? On pourrait dire que l'endroit où Husserl prend cette question à bras le corps, c'est dans les développements où il s'efforce de distinguer plusieurs ontologies matérielles — ou régionales — qui ont chacune leurs contraintes rationnelles spécifiques. Puisque, sur le plan apophantique, le problème de la compositionalité sémantique échappe à la logique formelle, il ne relève pas non plus, sur le plan ontologique, de l'ontologie formelle, mais bien de la classification des ontologies matérielles. En distinguant plusieurs types d'objectivité très différents, Husserl montre l'incongruité d'attribuer aux uns des qualités qui ne reviennent qu'aux autres, et ainsi de lier entre eux des matériaux qui n'ont pas "affaire les uns aux autres ». Et ce sont en fait ces ontologies régionales qui constituent, chez Husserl, le pendant ontologique des champs sémantiques. Or, il est clair que, sur ce plan ontologique, on ne peut évidemment reprocher à Husserl de n'avoir pas mené des investigations nombreuses, fouillées et minutieuses.

Mais ne peut-on pas lui reprocher d'avoir directement mené sur le seul plan ontologique ces investigations de la sphère de l'a priori matériel en

${ }^{1}$ Ibid., § 89a, p. 291-292.

${ }^{2}$ Ibid., $\S 89$ b, p. 294.

${ }^{3}$ Ibid., § 89b, p. 294. 
négligeant ce qui aurait d'abord dû être fait sur le plan apophantique, où les contraintes rationnelles visant à exclure le contresens auraient pu être distinguées de contraintes grammaticales préjudicielles visant à exclure le non-sens ? Selon cette objection, Husserl n'aurait pas, sur ce point, pris toute la mesure du tournant linguistique en philosophie et aurait opéré dogmatiquement sa division des ontologies matérielles plutôt que de la déduire de la sémantique comme l'ont par contre fait les philosophes du langage ordinaire. Lorsque Wittgenstein affirme que les notions de « couleur » ou de «nombre » doivent apparaître à la tête de chapitres de la grammaire philosophique ${ }^{1}$, il soutient que ce sont là des concepts qui définissent des groupes de significations de même « valeur », c'est-à-dire qui peuvent prendre les mêmes places et tenir les mêmes rôles dans un jeu de langage :

Quand l'enfant apprend «bleu est une couleur, rouge est une couleur, vert, jaune, tout cela ce sont des couleurs ", il n'apprend rien de neuf sur les couleurs, mais il apprend la signification d'une variable dans des propositions comme "l'image a de belles couleurs", etc. Une proposition de ce genre donne à l'enfant les valeurs d'une variable ${ }^{2}$.

Ces variables ont tout à la fois pour caractéristique de ne pouvoir recevoir que des déterminations d'un certain type - sous peine de non-sens - et de ne pouvoir, parmi les déterminations de ce type, en recevoir qu'une seule à la fois - sous peine de contresens ${ }^{3}$.

Mais, les catégories sont donc bien, pour Wittgenstein, des types de signification avant d'être des types d'être. Les catégories grammaticales ne sont pas la traduction des différences ontologiques; c'est au contraire la grammaire qui « dit quelle sorte d'objet » est chaque chose ${ }^{4}$. Husserl a-t-il pensé quelque chose de similaire?

Une manière de répondre à cette question serait d'identifier si la différence entre les types d'être relève, pour Husserl, de l'intention de signification ou de l'intuition. Qu'une idée ne puisse être verte ou dormir

${ }^{1}$ L. Wittgenstein, Remarques philosophiques (1930), op. cit., § 3, p. 53.

${ }^{2}$ Ibid., Ici encore, il en va de même pour le concept formel de «nombre », dont le Tractatus disait déjà : « Le concept de nombre n'est rien d'autre que ce qu'il y a de commun à tous les nombres, la forme générale du nombre. Le concept de nombre est le nombre variable » (Tractatus logico-philosophicus (1921), op. cit., § 6.022, p. 96. Cf. aussi Remarques philosophiques (1930), op. cit., § 110, p. 127).

${ }^{3}$ L. Wittgenstein, Remarques philosophiques (1930), op. cit., § 84, p. 108.

${ }^{4}$ L. Wittgenstein, Recherches philosophiques (1933-1949), op. cit., § 373, p. 171.

\section{1}


furieusement, qu'un nombre ne puisse être bleu ou se mouvoir dans l'espace, est-ce quelque chose qu'il convient de penser ou qu'il convient de voir? L'intuition est nécessaire pour prévenir le contresens matériel ; qu'un cercle ne puisse être carré, cela doit se voir. Mais la visée signitive ne suffit-elle pas à prévenir le non-sens matériel ; qu'un cercle ne puisse être anxieux, cela ne relève-t-il pas d'un a priori signitif préalable à tout a priori intuitif?

À cet égard, le propos des Idées directrices est un peu trompeur. Husserl y évoque en effet des éidétiques définissant des ontologies régionales et constituant autant de catégories matérielles après avoir revendiqué le caractère intuitif des essences ${ }^{1}$. Cette théorie de l'évidence, cependant, n'est pas le fin mot de la phénoménologie. Au contraire, Husserl dit explicitement à la fin du $\S 17$ que ces découpages régionaux intuitifs doivent être élucidés par des recherches phénoménologiques constitutives. Et, à cet égard, un rôle majeur est rendu à la visée signitive.

Développée dans la sixième Recherche logique, la théorie husserlienne de l'intuition comme remplissement d'intentions de signification accorde nécessairement une certaine priorité aux contraintes rationnelles propres à ces dernières. Et c'est pourquoi nombre de distinctions ontologiques ne reposent pas tant sur des différences dans la manière dont les objets se « donnent» effectivement que sur des différences dans la manière dont ils sont « visés ${ }^{2}$; deux objets ont des statuts ontologiques différents l'un de l'autre s'ils n'ont pas le même type de rapport intentionnel à la conscience. Parce qu'ils font l'objet de types fondamentalement différents de visées signitives, une chaise, un désir et un nombre sont des êtres différents. Pour Husserl, comme pour les philosophes analytiques, ce sont donc bien les types de visées de sens - les types sémantiques — qui dictent les types d'être les types ontologiques - et non le contraire ${ }^{3}$.

Dans la mesure où elle envisage chaque objet en tant qu'il est constitué dans des actes intentionnels de plus ou moins grande complexité qui sont

${ }^{1}$ E. Husserl, Idées directrices pour une phénoménologie (1933-1949), op. cit., § 16. Cf. aussi $\S \S 69$ sq. pour les sciences éidétiques fondées sur l'évidence.

${ }^{2}$ Jocelyn Benoist souligne que l'acte intentionnel est catégorial avant d'être rempli (Phénoménologie, sémantique, ontologie, op. cit., p. 135). Et l'intuition catégoriale n'est en fait rien d'autre que l'intuition sensible mais envisagée selon un mode nouveau, que définit une visée particulière (ibid., p. 141). Cf. aussi Denis Seron, Objet et signification, Paris, Vrin, 2003, p. 231 : «tel ceci peut être visé comme cheval, comme animal, comme corps physique, ...»

${ }^{3}$ Sur ce point, cf. mon article «Que le mode de donation dépend du monde de constitution: l'intuition des idéalités », in M. Maesschalk et R. Brisart (éds.), Idéalisme et phénoménologie, Hildesheim, Olms, 2007, p. 185-210.

\section{2}


eux-mêmes fondés sur des actes plus simples, toute la théorie phénoménologie de la constitution offre une sémantique avant même d'offrir une ontologie. Car les rapports de fondation entre intentions de signification président aux rapports de fondation entre actes intuitifs. C'est parce que, via un acte d'abstraction catégoriale, je vise, non pas ce triangle-ci dans sa singularité, mais à travers lui le triangle en général que peut m'apparaître une intuition non pas sensible mais catégoriale qui me fait voir sur lui un certain nombre de propriétés qui relèvent du triangle en général. Sans doute seule l'intuition catégoriale peut-elle me fournir les propriétés qu'ont effectivement les figures géométriques - et ainsi me livrer l'a priori matériel de la région ontologique correspondante - mais le type catégorial «figure géométrique » m'est déjà livré dans les intentions de signification qui en constituent le sens et dans la manière dont elles sont fondées sur des intentions de signification plus simples.

La théorie phénoménologique de la constitution est en fait le pendant exact du système logiciste de (re)construction logique du monde, à la différence près - mais qui est de taille - que Husserl ne croit pas que les rapports de fondation entre intentions de signification sont exclusivement logiques (au sens de la logique extensionnelle), et que peut donc être tracée d'emblée la carte parfaitement définie des types sémantiques possibles. Tous les objets de la science sont bien constitués les uns à partir des autres et in fine à partir des réalités matérielles ${ }^{1}$, mais les rapports qui président à cette fondation signitive ne se réduisent pas à ce que prévoit la seule théorie des ensembles. Reste que, dans son volet signitif préalable à tout intérêt pour les intuitions ainsi permises, la théorie phénoménologique de la constitution tout entière est au fond la grammaire matérielle que Husserl n'a pas explicitement théorisée en tant que telle. Parce qu'elle rapporte toute donation d'objet à des visées signitives, la phénoménologie constitutive fonde sa théorie de l'objet dans une grammaire ${ }^{2}$.

Contre cela, on fera peut-être valoir l'exemple classique de l'interdépendance entre étendue et couleur, interdépendance qui, d'après Husserl, ne pourrait être fournie par les intentions de signification mais seulement donnée par l'intuition. Que toute couleur doive être étendue et qu'à l'inverse toute étendue doive être colorée, n'est-ce pas là quelque chose qu'il faut voir et qui relève des contraintes de l'intuition et non seulement de l'intention de

${ }^{1}$ E. Husserl, Idées directrices pour une phénoménologie I, op. cit., § 152, trad. fr. p. 511.

${ }^{2}$ Sur ce point, Jocelyn Benoist ne manque pas de souligner l'avantage que prend Husserl sur Meinong (Phénoménologie, sémantique, ontologie, op. cit., p. 185-186). 
signification ? Rien, en effet, dans la visée de la signification «étendue » n'impose de rapport à la signification «couleur». Certes, mais à vrai dire l'idée d'une étendue sans couleur — ou l'énoncé qu'une certaine surface est dénuée de toute couleur - n'est pas un non-sens; les deux significations sont parfaitement séparables sur le plan intellectuel, et l'aveugle, ou n'importe quel sujet qui ferme les yeux, peut même parfaitement faire l'expérience de leur indépendance. Que, pour tous ceux qui voient comme nous, toute surface soit colorée, c'est certes une vérité intuitive, mais ce n'est précisément pas une contrainte grammaticale. Par contre, il y a sans doute quelque chose dans la constitution même de la signification " couleur » en tant que propriété qui est toujours inhérente à une surface mais qu'on peut, par abstraction, viser pour elle-même, qui prescrit un lien nécessaire à la signification "surface », et qui fait de "Toute couleur est étendue» un $a$ priori grammatical. Sans doute Husserl a-t-il raison de dire que le concept d'étendue n'est pas «analytiquement» inclus dans le concept de couleur ${ }^{1}$. Mais cela ne veut pas dire que le rapport n'est pas signitif, seulement que la fondation d'un acte de signification sur l'autre n'est pas purement logique.

Reste évidemment à déterminer le fondement de cette grammaire matérielle, comme d'ailleurs de la grammaire pure logique. Ce fondement est-il objectif (au sens de l'objectivisme sémantique de Bolzano)? subjectif (au sens de l'idéalisme transcendantal kantien) ? linguistique ? Husserl, on le sait, a hésité entre les deux premières conceptions. Mais il a toujours rejeté la dernière : l'a priori grammatical relève des significations elles-mêmes, pas de leur expression.

Dès les Recherches logiques, Husserl défendait le point de vue explicité dans le $\S 124$ des Idées directrices, selon lequel la couche de l'expression linguistique «n'est pas productive» mais qu'elle adopte l'essence de la couche sémantique qui reçoit l'expression ${ }^{2}$. Et c'est pourquoi, dans la quatrième des Recherches logiques, il insistait sur le caractère $a$ priori et non simplement inductif de la " grammaire universelle»:

Naturellement, on peut étendre l'idée de grammaire universelle au-delà de la sphère de l'a priori, en recourant à la sphère (vague à certains égards) de ce qui est universellement humain au sens empirique. Il peut et il doit y avoir une grammaire universelle dans ce sens le plus large et je suis (et ai toujours été) bien éloigné de mettre en doute que cette sphère plus vaste « ne soit riche en connaissances importantes et suffisamment déterminées ». Mais ici comme

${ }^{1}$ E. Husserl, Recherches logiques, III, op. cit., t. II, § 11, p. 37.

${ }^{2}$ E. Husserl, Idées directrices pour une phénoménologie I, op. cit., §124, trad. fr. p. 423 ; Morphologie apophantique $\S 134$. 
partout où des intérêts philosophiques sont en jeu, c'est une affaire de la plus haute importance que de séparer nettement l'apriorique de l'empirique et de reconnaître qu'à l'intérieur de cette discipline conçue dans toute son ampleur les connaissances intéressant le grammairien dans la morphologie des significations ont leur caractère propre, précisément, en tant qu'appartenant à une discipline apriorique qui doit être dégagée dans sa pureté ${ }^{1}$.

Héritier de l'objectivisme sémantique de Bolzano, Husserl revendiquait une fondation objective (c'est-à-dire dans les lois de combinaison des significations elles-mêmes) plutôt que linguistique de la grammaire. C'est là d'ailleurs ce qu'il réitérera dans Logique formelle et logique transcendantale :

Ce n'est pas sans raison que la morphologie des significations a été qualifiée dans mes Logische Untersuchungen de « grammaire pure logique ». Et en une certaine façon ce n'est pas non plus sans raison qu'il a été dit souvent que la logique formelle s'est laissée diriger par la grammaire. Mais pour la morphologie ce n'est pas un reproche, c'est au contraire nécessité, pourvu qu'au fait d'être dirigé par la grammaire (die Grammatik) (ce qui doit faire songer aux langages qui ont existé de fait et à leur description grammaticale) soit substitué le fait d'être dirigé par le grammatical (das Grammatische) luimême ${ }^{2}$.

Qu'elle soit formelle ou matérielle, la grammaire philosophique traduit des rapports entre les significations elles-mêmes. D'où la prudence avec laquelle il faut considérer l'intégration par Husserl du «tournant linguistique » de la philosophie contemporaine; si Husserl pratique sans conteste l'ascension sémantique, il ne donne pas pour autant dans l'idéalisme linguistique. Sans doute les classifications ontologiques relèvent-elles du sens plutôt que de l'intuition, mais cela ne veut pas dire qu'elles sont un pur produit de telle ou telle langue. La grammaire matérielle n'est pas la linguistique; c'est l'étude des rapports entre significations, rapport dont on a dit qu'ils ne sont pas euxmêmes exclusivement logiques ${ }^{3}$.

${ }^{1}$ Ibid., § 14, p. 132-133.

${ }^{2}$ E. Husserl, Logique formelle et logique transcendantale, op. cit., § 22, p. 98.

${ }^{3}$ En ce sens, comme le dit Jocelyn Benoist, la grammaire est elle-même une science régionale, science des rapports a priori qui régissent le domaine des significations (J. Benoist, L'a priori conceptuel : Bolzano, Husserl, Schlick, Paris, Vrin, 1999, p. 115-117). 